Southern Illinois University Carbondale

OpenSIUC

Articles

Department of Electrical and Computer

Engineering

Fall 12-20-2016

\title{
Auditory fatigue model applications to predict noise induced hearing loss in human and chinchilla
}

Pengfei Sun

Southern Illinois University Carbondale

Daniel Fox

Southern Illinois University School of Medicine

Kathleen Campbell

Southern Illinois University Springfield

Jun Qin

Southern Illinois University Carbondale, jqin@siu.edu

Follow this and additional works at: http:/ / opensiuc.lib.siu.edu/ece_articles

\section{Recommended Citation}

Sun, Pengfei, Fox, Daniel, Campbell, Kathleen and Qin, Jun. "Auditory fatigue model applications to predict noise induced hearing loss in human and chinchilla." Applied Acoustics 119, No. C (Fall 2016): 57-65. doi:10.1016/j.apacoust.2016.12.007.

This Article is brought to you for free and open access by the Department of Electrical and Computer Engineering at OpenSIUC. It has been accepted for inclusion in Articles by an authorized administrator of OpenSIUC. For more information, please contact opensiuc@lib.siu.edu. 


\title{
Auditory Fatigue Model Applications to Predict Noise Induced Hearing Loss in Human and Chinchilla
}

\author{
Pengfei Sun ${ }^{\mathrm{a}}$, Daniel Fox ${ }^{\mathrm{b}}$, Kathleen Campbell ${ }^{\mathrm{b}}$, Jun Qin ${ }^{\mathrm{a}, *}$ \\ ${ }^{a}$ Department of Electrical and Computer Engineering, Southern Illinois University, \\ Carbondale, Illinois, USA 62901 \\ ${ }^{b}$ Department of Medical Microbiology, Immunology, and Cell Biology, School of Medicine, \\ Southern Illinois University, Springfield, IL, 62794.
}

\begin{abstract}
Noise induced hearing loss (NIHL) remains a severe health problem worldwide. Current noise metrics and modeling have assessment limitations on gradually developing NIHL (GDNIHL). In this study, we applied a complex velocity level (CVL) auditory fatigue model to quantitatively assess the impact of basilar membrane (BM) movement on GDNIHL. The transfer functions of chinchilla and human auditory systems, including the triple-path nonlinear (TRNL) filters to simulate the inner ear responses, are applied to obtain BM velocity distribution. Chinchilla and human experimental hearing loss data are used to validate the proposed CVL model's effectiveness. The results reveal that the developed CVL model demonstrates high correlations with both chinchilla and human hearing loss data. The linear regression based correlation study indicates the proposed CVL model may accurately predict NIHL in both human and chinchilla.
\end{abstract}

Keywords: Noise induced hearing loss, auditory fatigue, hair cell loss, mammalian auditory filters

\footnotetext{
${ }^{*}$ Corresponding author

Email addresses: sunpengfei@siu.edu (Pengfei Sun), jqin@siu.edu (Jun Qin)
} 


\section{Introduction}

Noise induced hearing loss (NIHL) is one of the most common occupationally related diseases worldwide. Exposure to excessive noise is the major avoidable cause of permanent hearing loss [1]. Over 500 million people are still at risk of 5 developing NIHL globally [2]. Hearing loss lowers quality of life, impairs social interactions, causes isolation, and even causes loss of cognitive function. In terms of evolving time, NIHL can be briefly categorized into two types: acoustic trauma caused hearing loss and gradually developing NIHL (GDNIHL). Acoustic trauma often refers to a direct mechanical damage caused by short duration high intensity noise exposure (i.e., impulsive noise with peak sound pressure level (SPL) greater than $125 \mathrm{~dB}$ ). GDNIHL is progressive deterioration of the auditory periphery when long-time exposed to noise with SPL above $85 \mathrm{~dB}$ [3. Despite several factors (e.g., age, smoking, genetics, etc.) [2] may affect GDNIHL, the noise exposure is considered as the major cause of GDNIHL.

Several studies 4, 5, 6] show that NIHL can be considered as the mechanical failure of auditory system. Generally, conductive hearing loss and sensorineural hearing loss caused by noise exposure are presented as certain forms of mechanical malfunctions of auditory organs. For conductive hearing loss, concussion from explosion can lead to a disorder of middle ear, for instance, tympanic membrane perforations [7. Sensorineural hearing loss occurs when there is damage to the cochlear receptor organ, and the pathology can be found in the neural, sensory, and supporting cells of cochlea [3]. As one type of sensorineural hearing loss, GDNIHL is caused by multiple exposures to excessive noise, in which the stapes footplate hammer against the oval window of cochlea. The repeated flexing of the BM squeezes or stretches the outer hair cell (OHC) and the inner hair cell (IHC), and eventually cause hearing loss in cochlea.

International standards and regulations have been developed to estimate NIHL [8, 9, 10]. The noise metrics in these standards and regulations are developed with either waveform based empirical strategies (e.g., peak acoustic pres- 
$(\mathrm{EEH})\left(\right.$ e.g., A-weighted equivalent sound pressure level, $\left.L_{A e q}\right)$ [11, 12, 13]. Due to the lack of intrinsic study on the underlying mechanisms, these metrics are not sufficient enough to reveal the relation between the hearing loss and the noise exposure.

35 To better understand underlying mechanisms, an intuitive idea is to investigate the GDNIHL in auditory model (AM) framework. Such approach has three advantages: 1) transfer functions of AMs can vividly demonstrate the energy flow of noise; 2) AMs provide accurate evaluations on the ear's response to noise; 3) AMs can quantitatively describe the physical movements of hearing organs (e.g., vibrations of basilar member (BM)), which is strongly correlated with GDNIHL. In recent years, AMs has been used to develop more advanced models for the assessment and prediction of NIHL. Price proposed an auditory hazard assessment algorithm for human (AHAAH) model [14] to investigate acoustic trauma caused by high-level impulse noise (SPL $\geq 140 \mathrm{~dB})$. In another

45 study, Song introduced stapes velocity as an indication to predict impulse noise induced hearing loss incorporating an analog AM [15]. His model used the velocity of the stapes in the middle ear as input loads but did not involve with the auditory fatigue theory to predict of hearing loss.

For NIHL study, the key consideration for choosing an AM is how to accurately quantify the flow of acoustic energy from the environment into the inner ear. As a cascade filter model, triple-path nonlinear (TRNL) filter is an efficient model to simulate mammalian cochlea (e.g., human and chinchilla) [16]. TRNL filter, as the improved version of DRNL, is more flexible and accurate on describing the response of mammalian inner ear to stimulus, including both chinchilla and human beings. By introducing an extra all-pass parallel filter path, TRNL can reproduce the high-frequency plateau well. Hence, in our study, TRNL filter will be introduced to simulate the transfer function of mammalian inner ear and obtain the BM velocities.

$\mathrm{AM}$ is useful to describe the motions of BM responding to noise stimulus, 60 but has no indication on long-term integration effects of the noise hazardous. Considering that GDNIHL is the result of long-time accumulated hazardous, it 
can be approximately treated as the fatigue damage that happens to material structures [17, 18. Therefore, to quantitatively evaluate the long-term fatigue damage of GDNIHL, a corresponding fatigue model should be developed. The fundamental principle of fatigue models is based on material fatigue theories, including the S-N curve and the Miners rule. In S-N curve scenario, the complex loads on BM are treated as the a series of sinusoidal stresses, and each adjacent load counts to the functional failure independently. Therefore, the inputs on BM are classified based on their amplitude only. Comparatively, in Miner's rule, both amplitude and median value of the stimulus are taken into accounts - Instead of using BM displacement as the inputs [19, the BM velocity is translated as complex loads against the organ of Corti 20]. The rationality lies that BM velocity not only reflects the acoustic power flowing into the inner ear, but is also highly correlated with strain and loads [21, 22.

In this study, we expand our previous study [23, 24, by applying our developed complex velocity level (CVL) fatigue model to predict GDNIHL in both human and chinchilla. The generalized mammalian AM incorporating the triplepath nonlinear (TRNL) filter has been used to obtain BM velocities at different cochlea partitions (i.e., Equivalent Rectangular Band (ERB)). This generalized

so AM is adaptive to illustrate the auditory transfer functions of chinchilla and human by applying different parameters. Chinchilla and human experimental hearing loss data are applied to validate the proposed fatigue metric $L_{C V L}$ for the prediction of GDNIHL. The preferment of the proposed model is also compared to conventional noise metrics (e.g., $L_{A e q}$ and $L_{e q}$ ).

\section{Methods AND Materials}

\subsection{Transfer Functions of mammalian Auditory System}

Mammalian ear comprises external, middle, and inner ears. The coupled motion of tympanic membrane (TM), ossicles, and stapes footplate are the primary path for conducting environmental sound into the inner ear. 


\subsubsection{External ear and middle ear}

The primary function of the external ear and middle ear is to gather and conduct sound energy into the inner ear. The external ear likes a tube, with one end close by the tympanic membrane. The resonant characteristics of the external ear help determine the acoustic energy delivered to the cochlea. The middle

95 ear acts as an impedance-matching device that compensate the transmission loss when sound is introduced to the fluid-filled cochlea [25]. The phenomenon of middle ear muscle contraction (MEMC), known as an autonomic reflex that tightens the muscles of the middle ear, can affect the transfer function of middle ear [26]. MEMC is more frequently found in impulse or impact noise circumstances [3, 27. For generalized auditory system analysis in ambient noise, a fixed transfer function of middle ear should be used [28].

Figure.1 shows the transfer function of the external and middle ears of chinchilla [29] and human [30. In the external ear (Fig.1a), the transfer function of human is slightly shifted from that of chinchilla and the two transfer function shapes are almost the same. In the middle ear (Fig.1b), the human transfer function is different from chinchilla. The human has significantly higher gain than the chinchilla in middle frequency range $(500-5000 \mathrm{~Hz})$.

In this study, transfer function of the middle ear is characterized by stapes velocity transfer function (SVTF). The SVTF was defined as the ratio between linear velocity of stapes $V_{S}$ and sound pressure near the TM in the ear canal $P_{T M}\left(\mathrm{SVTF}=V_{S} / P_{T M}\right)$ [25], where the linear velocity $V_{S}$ could be obtained by dividing volume velocity $U_{S}$ by the average footplate area.

\subsubsection{Inner ear model-TRNL filter}

The cochlea is assumed to be a two-chambered, fluid-filled box with rigid side walls 19, and the partition between chambers is assumed rigid, excepting the flexible BM with elastic deformation. When the stapes motion produces pressure within the cochlea vestibule, sound stimulus can be transferred as BM vibrations [33. Several phenomenological models have been introduced to simulate movements over different sites along BM [32, 34]. 

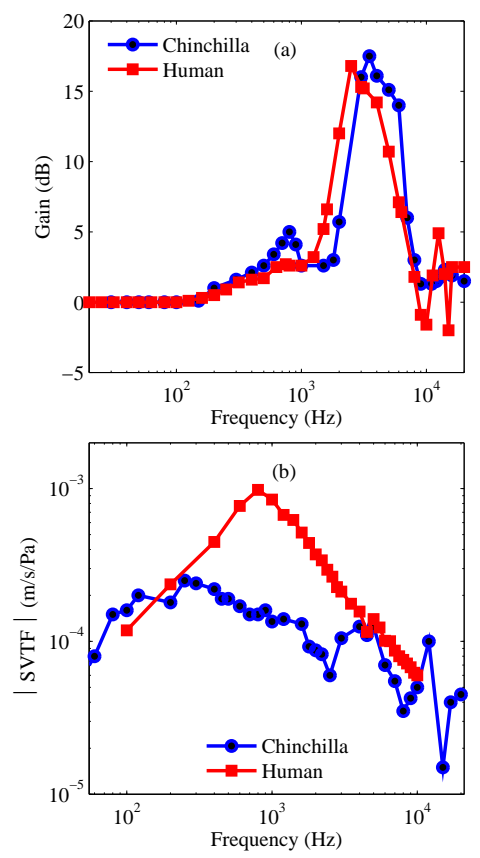

Figure 1: The transfer function of (a)the external ear and (b) the middle ear of chinchilla (blue) 31] and human (red) [32.

In this study, the TRNL filter [16] is utilized to obtain the BM responses along mammalian cochlea partitions. The input for TRNL filter is the linear velocity of stapes $V_{S}$ and the output represents the BM velocity of a particular location along the cochlea partition. Each individual site is represented as a tuned system with three parallel signal-processes paths 32 .

Each individual bandpass function is a cascade of two or more gammatone filters [35] with unit gain at center frequency $(\mathrm{CF})$. The low pass function comprises second-order low pass filters. In the nonlinear path, the CFs and bandwidths of the gammatone filters are the same. The compressive function shape in the nonlinear path is determined from animal data, and defined as

$$
y[t]=\operatorname{SIGN}(x[t]) \times \operatorname{MIN}\left(a|x[t]|, b|x[t]|^{c}\right)
$$

where $x(t)$ is the output of the first filter in the nonlinear path. $a, b$, and $c$ are parameters of the model. The parameter values in TRNL [32, 16] are 

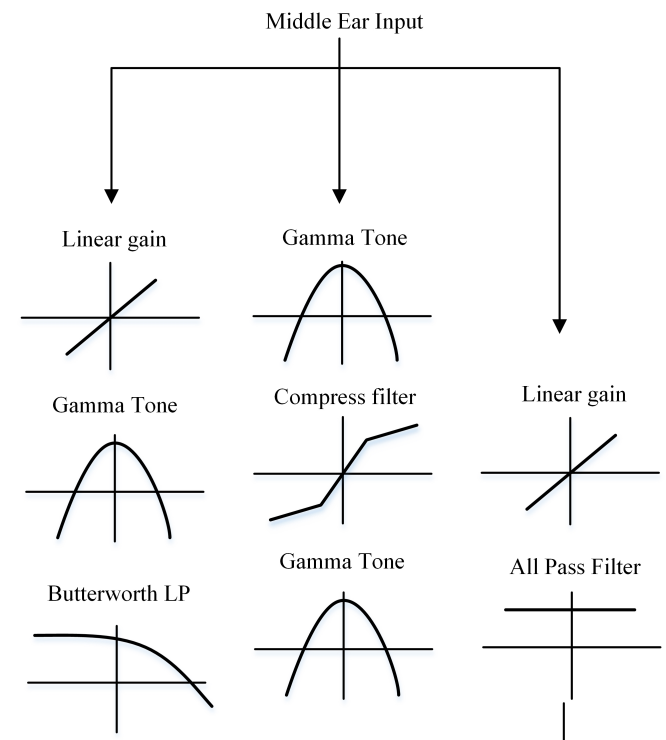

Gamma Tone

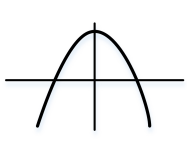

All Pass Filter

Butterworth LP
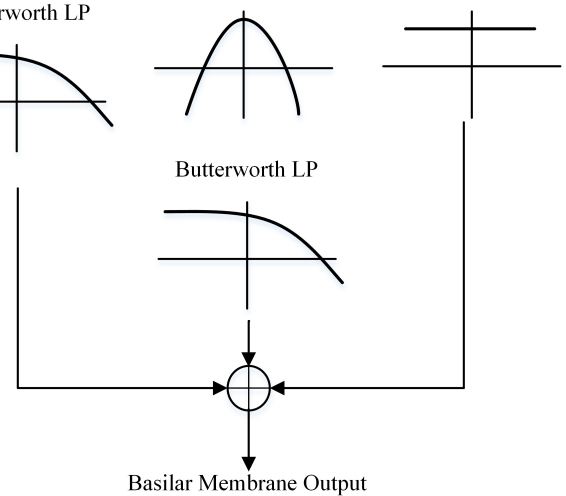

Figure 2: Schematic diagram of the TRNL filter, in which the velocities of stapes in middle ear are passed through three parallel branches to obtain the velocities of BM.

summarized in Table 1

\subsection{Basilar Membrane Velocity Based Fatigue Model CVL}

In our previous work [23, complex velocity level (CVL) based on the Miner's rule was proposed to calculate noise induced cumulative hazard. Miner's rule has been commonly used to predict materials high-cycle fatigue life under complex loads. Accordingly, the CVL model takes into account potential hearing loss correlated with both the amplitude transition and mean value of $\mathrm{BM}$ velocities. In a single $\mathrm{BM}$ vibration cycle, the instantaneous hearing fatigue in $\Delta t$ can be 
Table 1: Parameters of the TRNL filter which are used to simulate chinchilla 16 and human inner ear 32 .

\begin{tabular}{|c|c|c|c|c|c|c|c|c|c|c|c|c|c|}
\hline & \multicolumn{7}{|c|}{ Chinchilla } & \multicolumn{6}{|c|}{ Human } \\
\hline & $0.8 \mathrm{kHz}$ & $5.5 \mathrm{kHz}$ & $7.25 \mathrm{kHz}$ & $9.75 \mathrm{kHz}$ & $10 \mathrm{kHz}$ & $12 \mathrm{kHz}$ & $14 \mathrm{kHz}$ & $0.25 \mathrm{kHz}$ & $0.5 \mathrm{kHz}$ & $1 \mathrm{kHz}$ & $2 \mathrm{kHz}$ & $4 \mathrm{kHz}$ & $8 \mathrm{kHz}$ \\
\hline \multicolumn{14}{|c|}{$\mathrm{I}^{\prime}$} \\
\hline GT cascade & 5 & 5 & 5 & 5 & 5 & 5 & 5 & 2 & 2 & 2 & 2 & 2 & 2 \\
\hline LP cascade & 7 & 7 & 7 & 7 & 7 & 7 & 7 & 4 & 4 & 4 & 4 & 4 & 4 \\
\hline $\mathrm{CF}_{l i n}$ & 750 & 5000 & 7400 & 9000 & 9000 & 11000 & 13000 & 235 & 460 & 945 & 1895 & 3900 & 7450 \\
\hline $\mathrm{BW}_{\text {lin }}$ & 450 & 3000 & 2500 & 3000 & 3500 & 5000 & 4000 & 115 & 150 & 240 & 390 & 620 & 1550 \\
\hline $\mathrm{LP}_{\text {lin }}$ & 750 & 6000 & 7400 & 9000 & 8800 & 12000 & 13500 & 235 & 460 & 945 & 1895 & 3900 & 7450 \\
\hline Gain,g & 500 & 190 & 3000 & 300 & 500 & 500 & 350 & 1400 & 800 & 520 & 400 & 270 & 250 \\
\hline \multicolumn{14}{|l|}{ Nonlinear } \\
\hline GT cascade & 3 & 3 & 3 & 3 & 3 & 3 & 3 & 3 & 3 & 3 & 3 & 3 & 3 \\
\hline LP cascade & 4 & 4 & 4 & 4 & 4 & 4 & 4 & 3 & 3 & 3 & 3 & 3 & 3 \\
\hline $\mathrm{CF}_{l i n}$ & 730 & 5850 & 7800 & 9800 & 10000 & 12000 & 15000 & 250 & 500 & 1000 & 2000 & 4000 & 8000 \\
\hline $\mathrm{BW}_{l i n}$ & 350 & 1800 & 2275 & 1650 & 1800 & 2000 & 3200 & 84 & 103 & 175 & 300 & 560 & 1100 \\
\hline $\mathrm{LP}_{n l}$ & 730 & 5850 & 7800 & 9800 & 10000 & 12000 & 15000 & 250 & 500 & 1000 & 2000 & 4000 & 8000 \\
\hline Gain,a & 850 & 3000 & 15000 & 9000 & 15000 & 22500 & 3000 & 2124 & 4609 & 4598 & 9244 & 30274 & 76354 \\
\hline Gain,b & 0.03 & 0.04 & 0.06 & 0.05 & 0.06 & 0.07 & 0.045 & 0.45 & 0.28 & 0.13 & 0.078 & 0.060 & 0.035 \\
\hline Exponent,c & 0.25 & 0.25 & 0.25 & 0.25 & 0.25 & 0.25 & 0.25 & 0.25 & 0.25 & 0.25 & 0.25 & 0.25 & 0.25 \\
\hline \multicolumn{14}{|l|}{ Linear all-pass } \\
\hline Gain, k & 10 & 0.4 & 20 & 1 & 2 & 20 & 20 & - & - & - & - & - & - \\
\hline
\end{tabular}

quantitatively described by [23]

$$
\begin{aligned}
H_{V(t), \Delta t} & =\frac{\int_{\Delta t} V(t) d N(t)}{H_{0}} \\
& =\frac{\sum_{j}\left|V_{j}\right| \cdot N_{j}}{H_{0}}
\end{aligned}
$$

where the BM velocities $V(t)$ are regarded as complex stress, and $N(t)$ is the corresponding failure cycles at time $t$, respectively. In the discrete form, the subindex refers to $j$ th category of loads. $H_{0}$ is the hearing loss at the ERB with $1 \mathrm{kHz} \mathrm{CF}$.

In practice, occupational noise should be considered as complex and often random loads. Thus, the response BM velocities also demonstrate complex distribution. In the CVL model, the 2-dimensional histogram of input loads (i.e., the velocities of BM) (Fig.3a) is obtained in terms of differential velocity $V_{\text {Amplitude }}$ and mean velocity $V_{\text {mean }}$ in the time domain (Fig.3(b)) [23]. Based on the histogram of complex input loads, respecting to both $V_{\text {Amplitude }}$ and $V_{\text {mean }}$ values, the hearing loss $H_{i, C V L}$ is the integration of different categories 
of inputs along the time axis as

$$
H_{i, C V L}=\sum_{j \subset K} N_{j} \cdot\left|V_{\text {amplitude }}(i, j) \cdot V_{\text {mean }}(i, j)\right|
$$

where $K$ is the total number of load categories, including $j$ th type velocity, and $i$ is the ERB band.

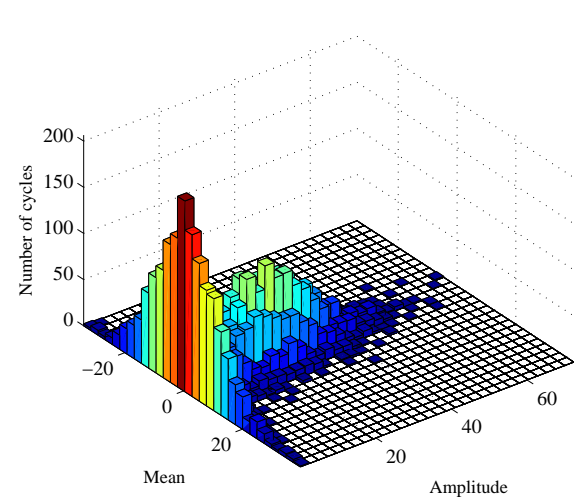

(a)

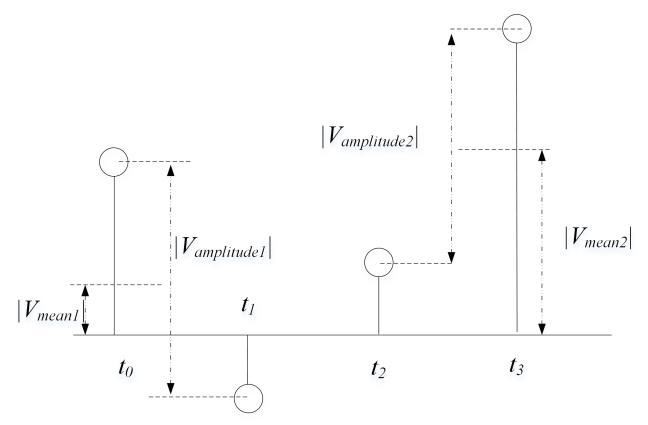

(b)

Figure 3: Histogram of BM velocities (a) categorized according to the transition amplitude and mean values of velocities as (b) at the $i$ th ERB in 1 second. be obtained

$$
\begin{gathered}
L_{i, C V L}=10 \log 10\left(\frac{H_{i, C V L}^{2}}{H_{0}^{2}}\right) \\
L_{I, C V L}=10 \log 10\left(\frac{\sum_{i \subset I} H_{i, C V L}^{2}}{H_{0}^{2}}\right)
\end{gathered}
$$

where $L_{i, C V L}$ and $L_{I, C V L}$ are the $\log$ scale hearing loss metrics at $i$ th ERB and $I$ th frequency band. $L_{i, C V L}$ reflects the integration of hearing loss level at the $i$ th ERB temporally. Comparatively, $L_{I, C V L}$ is the hearing loss level in frequency band $I$, in which several ERBs might be included. Based on the CVL model,(4) and (5) can be used to assess the auditory risk of hazardous caused by the BM vibration. 


\subsection{Experimental Data}

165

sure data in the lab and human hearing loss data measured in the fields, were provided by a research group at State University of New York at Plattsburgh. The chinchilla data were used in published animal noise exposure experiments [36, 37. The human hearing loss data were also published in recent works

\subsubsection{Chinchilla noise exposure data}

Chinchilla noise exposure data were used for validation of the proposed fatigue metric. Two hundred seventy three chinchilla in 22 groups were exposed to certain noise types for five-consecutive-days at 24 hours per day. chinchilla 3 Gaussian noises with 90, 95, and $100 \mathrm{dBA}$, and 19 complex noises (one at $95 \mathrm{dBA}$, two at $90 \mathrm{dBA}$, and 16 at $100 \mathrm{dBA}$ ) 36, 37. The digitally recorded noise samples (320 sec for each noise sample) [40] are used for noise analysis in this study. Detailed descriptions of the noise data and experimental protocols of animal studies are available in previous publications [36, 37.

In the animal noise exposure experiments, both permanent threshold shift (PTS) and temporary threshold shift (TTS) were determined at 0.5, 1, 2, 4, 8, and $16 \mathrm{kHz}$ for each animal as summarized in Table 2. In addition, to assess the overall hearing loss in whole frequency range, the averaged effective PTS has

\subsubsection{Human hearing loss data}

The human hearing loss data comprised Gaussian and non-Gaussian industrial noise recordings and hearing levels from workers in two noisy industrial 
Table 2: PTS and TTS of Chinchilla at different center frequencies of octave bands for different noise exposures.

\begin{tabular}{ccccccccccccccc}
\hline \multirow{2}{*}{ Samples } & \multirow{2}{*}{ Leq } & \multicolumn{1}{c}{ PTS } & \multicolumn{1}{c}{ TTS } \\
\cline { 3 - 9 } & & $0.5 \mathrm{kHz}$ & $1 \mathrm{kHz}$ & $2 \mathrm{kHz}$ & $4 \mathrm{kHz}$ & $8 \mathrm{kHz}$ & $16 \mathrm{kHz}$ & $0.5 \mathrm{kHz}$ & $1 \mathrm{kHz}$ & $2 \mathrm{kHz}$ & $4 \mathrm{kHz}$ & $8 \mathrm{kHz}$ & $16 \mathrm{kHz}$ \\
\hline G44 & 100.6 & 17.1 & 26.2 & 39.4 & 42.9 & 46.5 & 43.7 & 58.6 & 70.1 & 79.3 & 85.4 & 85.8 & 70.6 \\
G49 & 101 & 22.1 & 34.3 & 47.2 & 54.6 & 46.8 & 47.2 & 62.6 & 75.3 & 77.6 & 86.5 & 79.9 & 70.6 \\
G50 & 100.5 & 7.7 & 10.1 & 8.0 & 15.8 & 14.1 & 17.7 & 37.2 & 57.6 & 63.4 & 76.1 & 79.8 & 69.2 \\
G51 & 100.1 & 15.7 & 19.5 & 29.0 & 24.3 & 27.8 & 25.1 & 59.7 & 63.9 & 73.2 & 75.9 & 81.9 & 67.9 \\
G52 & 101.7 & 18.5 & 24.5 & 36.8 & 32.9 & 28.3 & 23.3 & 63.9 & 72.4 & 76.4 & 81.2 & 80.1 & 69.6 \\
G53 & 100.6 & 19.0 & 24.4 & 34.5 & 31.7 & 29.9 & 28.1 & 59.4 & 68.0 & 77.4 & 85.0 & 84.3 & 69.0 \\
G54 & 100.6 & 16.2 & 18.5 & 29.9 & 31.4 & 25.4 & 29.1 & 55.7 & 65.3 & 75.6 & 82.5 & 80.0 & 66.3 \\
G55 & 100.1 & 18.8 & 21.7 & 36.5 & 46.8 & 60.1 & 47.5 & 67.1 & 74.1 & 76.2 & 82.3 & 80.3 & 68.8 \\
G60 & 100.2 & 20.7 & 27.8 & 34.1 & 34.1 & 29.3 & 27.8 & 59.3 & 68.4 & 70.8 & 75.7 & 75.9 & 65.2 \\
G61 & 99.6 & 2.6 & 5.0 & 10.0 & 20.5 & 18.2 & 24.0 & 36.1 & 45.6 & 50.4 & 74.4 & 80.4 & 72.0 \\
G63 & 99.6 & 25.4 & 31.4 & 43.8 & 36.2 & 32.3 & 28.9 & 63.4 & 69.8 & 76.2 & 76.4 & 73.4 & 65.0 \\
G64 & 101.1 & 15.8 & 17.4 & 24.7 & 22.1 & 19.0 & 13.5 & 60.0 & 66.3 & 73.8 & 79.4 & 73.9 & 67.1 \\
G65 & 99.7 & 17.2 & 14.4 & 25.0 & 39.6 & 49.5 & 48.3 & 62.5 & 62.8 & 68.1 & 74.4 & 75.8 & 70.7 \\
G66 & 100.7 & 7.5 & 9.3 & 19.2 & 32.9 & 44.8 & 36.2 & 49.4 & 58.9 & 70.0 & 82.9 & 76.1 & 70.4 \\
G68 & 99.7 & 12.9 & 13.9 & 21.7 & 39.7 & 47.3 & 47.3 & 65.9 & 69.2 & 71.1 & 81.1 & 75.0 & 73.3 \\
G69 & 101 & 4.8 & 10.9 & 9.3 & 11.3 & 5.5 & 8.0 & 28.8 & 47.4 & 48.8 & 49.3 & 47.8 & 50.1 \\
G70 & 100.7 & 12.1 & 17.9 & 27.6 & 43.2 & 30.4 & 35.1 & 59.9 & 69.9 & 75.0 & 84.8 & 76.8 & 71.0 \\
G47 & 89.4 & 0.3 & -0.3 & 3.6 & 1.5 & 7.9 & 7.0 & 22.5 & 35.0 & 43.3 & 60.8 & 68.7 & 61.2 \\
G48 & 91.7 & 3.0 & 6.8 & 9.4 & 5.4 & 11.2 & 10.8 & 26.9 & 35.9 & 37.6 & 41.5 & 58.0 & 63.9 \\
G56 & 91.3 & 2.9 & 1.7 & 4.5 & 8.9 & 14.7 & 8.9 & 29.5 & 30.5 & 29.2 & 39.3 & 52.0 & 50.9 \\
G57 & 94.2 & 6.8 & 5.8 & 6.7 & 16.7 & 23.3 & 18.9 & 35.5 & 41.4 & 52.1 & 66.4 & 71.8 & 66.0 \\
G58 & 95.6 & 7.8 & 8.8 & 18.9 & 17.5 & 15.0 & 17.9 & 44.5 & 50.3 & 59.1 & 62.1 & 62.1 & 63.6 \\
\hline
\end{tabular}

environments [38, 39]. All participants were required to satisfy the following four criteria: (1) a minimum of at least 1-yr employment at the current task, (2) no history of genetic or drug-related hearing loss, head trauma, or ear diseases, (3) no military service, shooting activities, or high intensity nonindustrial noise exposure, and (4) no history of hearing protection use. A total of 195 workers out of 220 participants from two industries met the study criteria. Thirty-two of the participants were exposed to non-Gaussian noise for an average of $12.3 \pm 7.1$ yrs in a metal fabrication factory. The remaining 163 participants were exposed to a continuous Gaussian noise for an average $12.7 \pm 8.4 \mathrm{yrs}$ in a textile mill.

Each enrolled participant was given a general physical and an otologic examination. Pure air tone, conduction hearing threshold levels (HTLs) at 0.5, 1.0, $2.0,3.0,4.0,6.0$, and $8.0 \mathrm{kHz}$ were measured by an experienced physician for right and left ears. HTLs at each frequency were adjusted for age and gender using the 50th percentile values found in the standard [8] Annex B. Real-time noise signals (5-min duration) were recorded at the level of the participants ear. Nineteen noise signals from the non-Gaussian noise environment and 20 from the Gaussian noise environment were recorded with 16-bit resolution and 11 
105 dBA were used for noise analysis in this study (Table 3). The Nyquist limit $(5.5 \mathrm{kHz})$ limited PTS analysis to no more than a frequency band centered at $6 \mathrm{kHz}$. As a result, the $8 \mathrm{kHz}$ PTS data is not presented in Table 3 . In this study, the PTS collected at frequency band with $6 \mathrm{kHz} \mathrm{CF}$ has been processed CF. In addition, the averaged PTS, including $P T S_{1234}$ and $P T S_{2345}$ are used to evaluate the overall NIHL.

Table 3: PTS loss of Human at different frequency bands for different noise exposures.

\begin{tabular}{|c|c|c|c|c|c|c|c|c|c|c|c|c|c|}
\hline \multirow{2}{*}{ Samples } & \multirow{2}{*}{$L_{A e q}$} & \multicolumn{6}{|c|}{ Right Ear } & \multicolumn{6}{|c|}{ Left Ear } \\
\hline & & $0.5 \mathrm{kHz}$ & $1 \mathrm{kHz}$ & $2 \mathrm{kHz}$ & $3 \mathrm{kHz}$ & $4 \mathrm{kHz}$ & $6 \mathrm{kHz}$ & $0.5 \mathrm{kHz}$ & $1 \mathrm{kHz}$ & $2 \mathrm{kHz}$ & $3 \mathrm{kHz}$ & $4 \mathrm{kHz}$ & $6 \mathrm{kHz}$ \\
\hline Gm5c1a & 98.7 & 14.3 & 11.8 & 16.1 & 28.5 & 32.5 & 24.8 & 17.3 & 13.8 & 12.1 & 28 & 33 & 26.3 \\
\hline $\mathrm{Gm} 5 \mathrm{c} 1 \mathrm{~b}$ & 96.1 & 14.1 & 12.2 & 12.2 & 19.3 & 19.5 & 16.2 & 12.9 & 11.8 & 10.7 & 12 & 15.2 & 19.6 \\
\hline $\mathrm{Gm} 5 \mathrm{c} 2 \mathrm{a}$ & 9 & 13.6 & 13.6 & 12.2 & 9.9 & 14.8 & 17.7 & 16.1 & 13.6 & 11.2 & 9.9 & 14.8 & 12.2 \\
\hline $\mathrm{Gm} 5 \mathrm{c} 2 \mathrm{~b}$ & 104.5 & 16.5 & 17.1 & 13.4 & 15.5 & 16.4 & 16.5 & 15.3 & 14 & 12.8 & 16.8 & 16.4 & 16.5 \\
\hline Gm5c3a & 03 & 16.3 & 16.3 & 21.4 & 27 & 23.3 & 26.4 & 16.3 & 12.7 & 13.6 & 22.7 & 23.3 & 30 \\
\hline $\mathrm{Gm} 5 \mathrm{c} 3 \mathrm{~b}$ & 100.6 & 15.3 & 16.7 & 23.7 & 32.9 & 34.1 & 42.7 & 15.3 & 13.9 & 18.7 & 32.9 & 32 & 29.1 \\
\hline $\mathrm{Gm} 5 \mathrm{c} 4 \mathrm{a}$ & 95.5 & 14.4 & 17.9 & 16.1 & 28.2 & 31.4 & 35.6 & 15.8 & 15.8 & 16.1 & 24.3 & 30.3 & 25.9 \\
\hline $\mathrm{Gm} 5 \mathrm{c} 4 \mathrm{~b}$ & 105.6 & 15.1 & 15.1 & 16.2 & 32.9 & 40.3 & 36.7 & 16.3 & 13.8 & 14.9 & 27.1 & 40.7 & 37.1 \\
\hline $5 c 5 a$ & 9 & 10.4 & 10.4 & 9 & 14.3 & 13.7 & 10.9 & 13.3 & 10.4 & 8.3 & 7.9 & 13.7 & 15.1 \\
\hline $\mathrm{Gm}$ & 100.3 & 12.1 & 12.5 & 19.7 & 25.1 & 30.7 & 30 & 16.6 & 13.5 & 19.7 & 26.5 & 34.8 & 31.4 \\
\hline & 9 & 18.7 & 17.3 & 25.7 & 38.7 & 43 & 40.7 & 18 & 15.1 & 18.6 & 30.1 & 35.1 & 43.6 \\
\hline $\mathrm{Gm}$ & 101.1 & 13.6 & 12.3 & 9.2 & 11.3 & 17.4 & 19.9 & 13.6 & 12.1 & 8.0 & 12.3 & 21.9 & 21.7 \\
\hline & & 15.2 & 13 & 11.6 & 23.4 & 34.4 & 35.3 & 16.9 & 14.7 & 12.7 & 24 & 38.9 & 28.7 \\
\hline Tcc6a & 95.2 & 12.9 & 10.4 & 9.9 & 12.9 & 12.9 & 17.6 & 11 & 10.4 & 8 & 12.3 & 11.6 & 18.9 \\
\hline
\end{tabular}

\section{RESULTS AND DISCUSSIONS}

3.1. BM Velocity Distributions Obtained by the Chinchilla and Human Auditory Models

Four experimental noise samples (i.e., G63 and G61 from chinchilla, and Tcc1a and Gm5c2a from human) were used as inputs to test the AMs of chinchilla and human. The time-frequency (T-F) presentation of the BM velocity distributions are shown in Fig.4. The noise sample G63 simulates an impulsive noise (Fig.4(a)), while the sample G61 is a typical Gaussian continuous noise (Fig.4(b)). The recorded metal fabrication factory noise sample Tccla is a nonGaussian noise (Fig.4(c)). Comparatively, the noise sample Gm5c2a (Fig.4(d)) collected from textile mill is a Gaussian noise. 

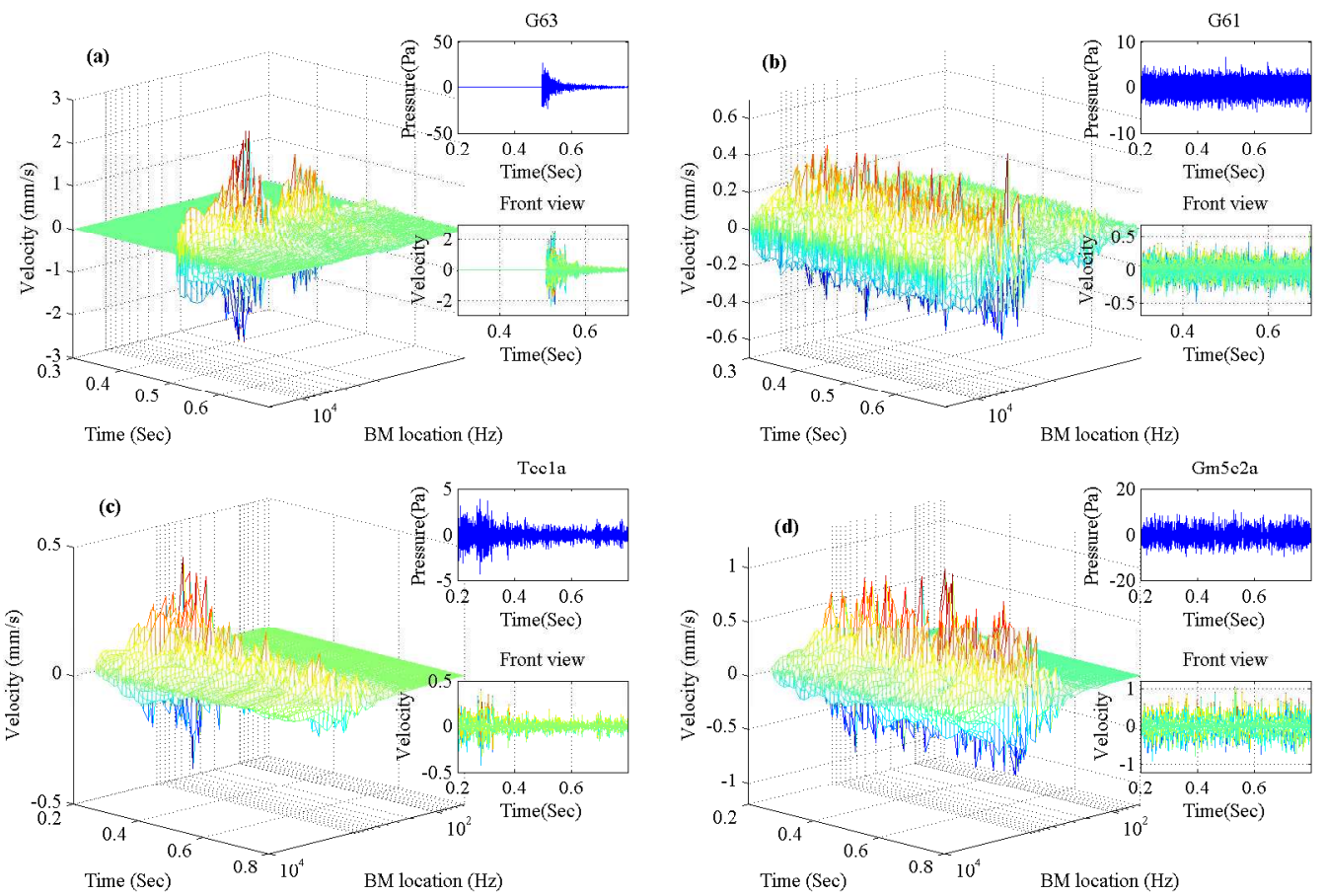

Figure 4: Time-frequency presentations of the BM velocities obtained by the developed chinchilla and human auditory models, responding to the experimental noise samples (a)G63,(b)G61, (c)Tcc1a, and (d)Gm5c2a. The partial waveforms of G63, G61, Tcc1a, and Gm5c2a in $0.6 \mathrm{sec}$ are shown in the top insert figures. The front views of the distributions of the $\mathrm{BM}$ velocities are shown in the bottom insert figures.

The distributions of the BM velocities along the time axis can accurately reflect the waveform of original noise signals (Figure 4 a-d front views). The AMs accurately transfer the acoustic pressure to the BM velocities in both chinchilla and human models. Furthermore, for chinchilla, the distributions of the BM velocities along the frequency axis are concentrated in the high frequency bands 235 (Fig.4(a),(b)). The BM velocities in the low frequency bands were significantly reduced by the chinchilla external-middle ear transfer function gain (as shown in Fig.1), which demonstrates a strong decayed gain at low frequency range. In contrast, for human (as shown in Fig.4(c) and 4(d)), the BM velocities are concentrated within frequency range of 500-5000 Hz. This is consistent with the transfer function of human middle ear (as shown in Fig.1(b)), which has significantly higher gain than the corresponding transfer function of chinchilla. 

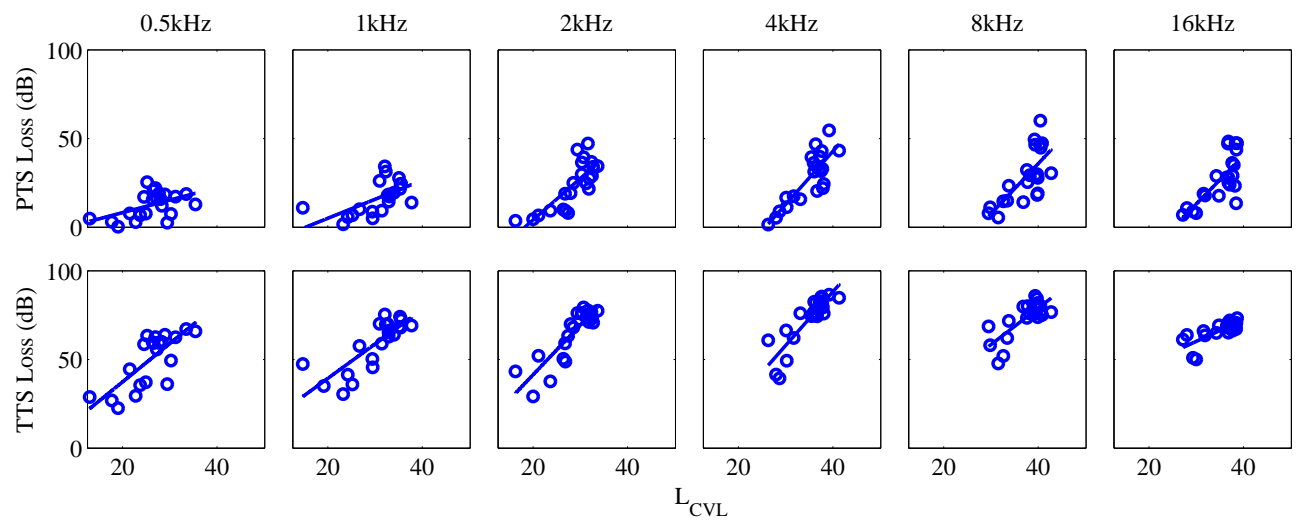

Figure 5: Scatting plots and fitting lines of pairs of the developed fatigue metrics $L_{C V L}$, and hearing loss indications, TTS and PTS at six octave frequency bands, averaged by all 22 groups of animal experimental data.

\subsection{Validation of the Developed CVL Fatigue Models in Chinchilla}

\subsubsection{Linear regression analysis at six octave bands}

The linear regression analysis of the developed fatigue metric $\left(L_{C V L}\right)$, and two hearing loss indications (PTS and TTS) at six frequency bands were conducted using all 22 chinchilla noise exposure groups. Figure 5 shows the fitting lines and scatting plots of the pairs of $L_{C V L}$ and the hearing loss indices. $L_{C V L}$ is calculated using a 40-sec time window. Each symbol in Fig.5 refers to a pair of a fatigue metric and an animal hearing loss index. The lines indicate the fitting results of the distributions of symbols. Six octave frequency bands centered at $0.5,1,2,4,8$, and $16 \mathrm{kHz}$ cover the $\mathrm{BM}$ frequency range.

Moreover, the linear correlation value $\left(r^{2}\right)$ of different metrics, including $L_{A e q}, L_{e q}$ and the proposed $L_{C V L}$, showed that in each octave frequency band, $L_{C V L}$ achieves the higher correlation with both PTS and TTS compared to the two conventional $L_{A e q}$ and $L_{e q}$ metrics (Table 44. $L_{C V L}$ has strong correlations with hearing loss indices at frequency bands centered at 1, 2, 4, and $8 \mathrm{kHz}$ than 0.5 and $16 \mathrm{kHz}$ because with higher $r^{2}$ values. In addition, $L_{C V L}$ showed the higher correlations with TTS than PTS at all frequency bands. TTS refers to the instant hearing loss immediately after a noise exposure, while the PTS is the permanent hearing loss after noise exposure with certain recovery time [6]. TTS 
directly reflects the mechanical failure caused by noise exposure. The developed fatigue models are based on the $\mathrm{BM}$ velocity, which reflects the mechanical vibration of $\mathrm{BM}$ in cochlea. Therefore, it is reasonable that the proposed fatigue metrics have higher correlations with TTS.

Table 4: Regression analysis of two hearing loss indices of chinchilla and different metrics at six octave bands centered at $0.5,1,2,4,8$, and $16 \mathrm{kHz}$

\begin{tabular}{lcccccc}
\hline \hline $\mathrm{r}^{2}$ & $0.5 \mathrm{kHz}$ & $1 \mathrm{kHz}$ & $2 \mathrm{kHz}$ & $4 \mathrm{kHz}$ & $8 \mathrm{kHz}$ & $16 \mathrm{kHz}$ \\
\hline$L_{C V L}$-PTS & $\mathbf{0 . 2 5}$ & $\mathbf{0 . 4 1}$ & $\mathbf{0 . 7 7}$ & $\mathbf{0 . 7 1}$ & $\mathbf{0 . 5 2}$ & $\mathbf{0 . 5 5}$ \\
$L_{A e q}-\mathrm{PTS}$ & 0.09 & 0.39 & 0.25 & 0.53 & 0.32 & 0.15 \\
$L_{e q}$-PTS & 0.07 & 0.37 & 0.25 & 0.54 & 0.30 & 0.12 \\
$L_{C V L}-\mathrm{TTS}$ & $\mathbf{0 . 5 7}$ & $\mathbf{0 . 6 5}$ & $\mathbf{0 . 8 1}$ & $\mathbf{0 . 7 6}$ & $\mathbf{0 . 6 5}$ & $\mathbf{0 . 5 7}$ \\
$L_{A e q}-\mathrm{TTS}$ & 0.29 & 0.57 & 0.41 & 0.60 & 0.50 & 0.51 \\
$L_{e q}-\mathrm{TTS}$ & 0.27 & 0.54 & 0.40 & 0.61 & 0.52 & 0.49 \\
\hline
\end{tabular}
spect to $T T S_{1248}$ and $P T S_{1248}$ show significantly higher correlations between $L_{C V L}$ and both hearing loss indications compared to $L_{A e q}$ and $L_{e q}$. Thus, the considerably high correlations between the $L_{C V L}$ and hearing loss data suggests that the proposed CVL model may accurately predict GDNIHL in chinchilla.

\subsection{Validation of CVL Models in Human}

The same procedure was implemented to conduct the linear regression analysis of $L_{C V L}$ and PTS at six frequency bands centered at $0.5,1,2,3,4$, and 5 

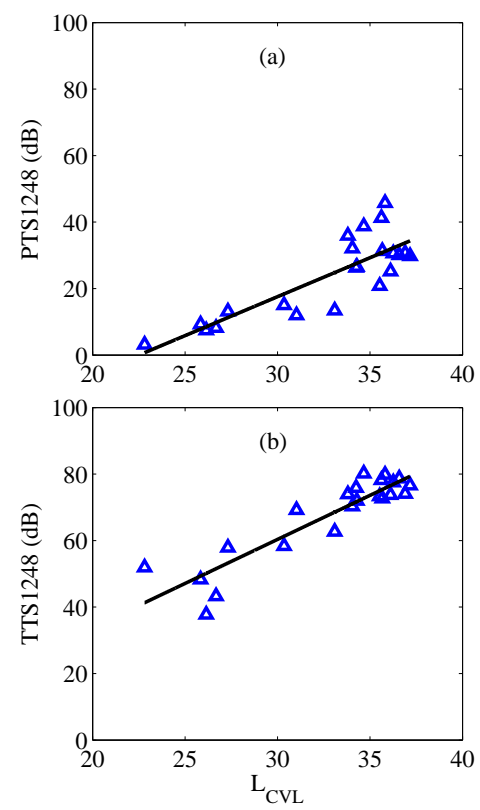

Figure 6: Regression analysis of averaged $P T S_{1248}$ and $T T S_{1248}$ of chinchilla and proposed fatigue metric $L_{C V L}$ with $R^{2}$ value 0.7 (a) and 0.82 (b), respectively.

Table 5: Regression analysis of two hearing loss indices of chinchilla and different metrics at averaged octave bands

\begin{tabular}{ccc}
\hline \hline & & \\
& $\mathrm{PTS}_{1248}$ & $\mathrm{TTS}_{1248}$ \\
\hline & & \\
$L_{C V L}$ & $\mathbf{0 . 7}$ & $\mathbf{0 . 8 2}$ \\
$L_{A e q}$ & 0.48 & 0.62 \\
$L_{e q}$ & 0.47 & 0.59 \\
\hline
\end{tabular}

kHz with 195 human participants.

In Fig.7, the horizontal coordinates of the 14 dots represent the $L_{C V L}$ of 14 occupational noise samples in each frequency band, whereas the vertical coordinates of the 14 dots in each sub-figure reflects the averaged PTS of participants exposed to certain noise samples. $L_{C V L}$ has a strong correlation with PTS in the $2,3,4$, and $5 \mathrm{kHz}$ frequency bands. $L_{C V L}$ is also linearly correlated with the PTS in all the frequency bands and peaks at 0.72 in the $5 \mathrm{kHz}$ frequency band (Table 6). In addition, $L_{C V L}$ has significantly higher $r^{2}$ values in all six frequency bands compared to $L_{A e q}$ and $L_{e q}$. Results indicate the $L_{C V L}$ metric 
Table 6: Regression analysis of PTS of human and different metrics at six frequency bands centered at $0.5,1,2,3,4$, and $5 \mathrm{kHz}$

\begin{tabular}{ccccccc}
\hline \hline $\mathrm{r}^{2}$ & $0.5 \mathrm{kHz}$ & $1 \mathrm{kHz}$ & $2 \mathrm{kHz}$ & $3 \mathrm{kHz}$ & $4 \mathrm{kHz}$ & $5 \mathrm{kHz}$ \\
\hline $\mathrm{L}_{C V L}-P T S$ & $\mathbf{0 . 3 8}$ & $\mathbf{0 . 4 0}$ & $\mathbf{0 . 6 2}$ & $\mathbf{0 . 5 9}$ & $\mathbf{0 . 6 8}$ & $\mathbf{0 . 7 2}$ \\
$\mathrm{L}_{\text {Aeq }}-P T S$ & 0.08 & 0.13 & 0.51 & 0.44 & 0.42 & 0.32 \\
$\mathrm{~L}_{\text {eq }}-P T S$ & 0.09 & 0.11 & 0.49 & 0.47 & 0.41 & 0.30 \\
\hline
\end{tabular}

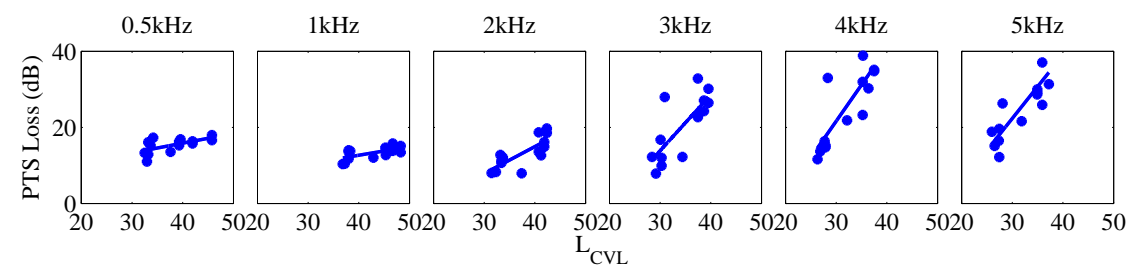

Figure 7: Scatting plots and fitting lines of pairs of the developed fatigue metric, $L_{C V L}$, and hearing loss indication (i.e., PTS) at six frequency bands, averaged by all of human hearing loss data in each group.

may be able to more accurately predict NIHL than conventional noise metrics, e.g., $L_{A e q}$ and $L_{e q}$.

Moreover, two averaged hearing loss indices $P T S_{1234}$ and $P T S_{2345}$ were used to validate the effectiveness of $L_{C V L}$ in the prediction of overall hearing loss in human. $L_{C V L}$ is linearly correlated with human hearing loss data (Fig.8) and the metric $L_{C V L}$ measured higher $r^{2}$ values than both $L_{A e q}$ and $L_{e q}$ (Table 7 ). Results indicate the proposed CVL model may accurately predict the GDNIHL caused by occupational noise (including both Gaussian an non-Gaussian noise) in human.

Table 7: Regression analysis of two hearing loss indices (PTS 1234 and $\left.P T S_{2345}\right)$ of human and different metrics at averaged octave bands

\begin{tabular}{ccc}
\hline \hline & $\mathrm{PTS}_{1234}$ & $\mathrm{TTS}_{2345}$ \\
\hline$L_{C V L}$ & $\mathbf{0 . 7}$ & $\mathbf{0 . 7 4}$ \\
$L_{\text {Aeq }}$ & 0.56 & 0.57 \\
$L_{e q}$ & 0.54 & 0.49 \\
\hline
\end{tabular}

The hearing damage calculated by the proposed auditory fatigue model accu- 

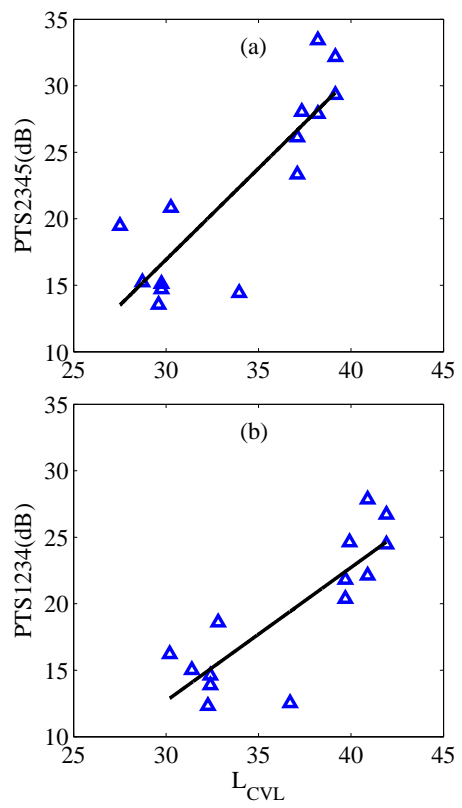

Figure 8: Scatting plots and fitting lines of (a) $L_{C V L}$ vs. $P T S_{2345}$ and (b) $L_{C V L}$ vs. PT $S_{1234}$ averaged by all each group of human hearing loss data.

rately fits the hearing loss data for both chinchilla and human. It strengthens the conclusion that the hearing loss is a reflection of auditory fatigue phenomenon. In addition, the chinchilla has been the most common animal model for noise induced hearing loss (NIHL) in human with 221 articles published since 1971 [4]. Regardless of the study target tissue in the various studies, the noise exposure must first pass through the cochlea. Thus the information in this study may indicate that the commonly used chinchilla model is appropriate to translate research in NIHL to human.

\section{Conclusion}

In this study, an auditory fatigue model (i.e., CVL model) was tested as a predictor of occupational noise-induced GDNIHL for both chinchilla and human. The mammalian AM was introduced by combining the TRNL filter with external-middle ear transfer function to accurately characterize BM vibration. 
Both animal noise exposure data and human field hearing loss data were used to validate the effectiveness of the developed CVL model. Results indicated the developed CVL model demonstrated high correlations with experimental hearing loss data in both chinchilla and human. The proposed metric $L_{C V L}$ also showed significant advantage on hearing loss prediction compared to $L_{A e q}$

320 and $L_{e q}$. Results indicate that the developed model may accurately predict the NIHL in both chinchilla and human. The developed CVL model may be applied for human NIHL assessment in various industrial and military applications.

\section{Acknowledgment}

This research was supported in part by National Institute on Deafness and 325 Other Communication Disorders (NIDCD)(R01 DC014549-01), and Illinois Clean Coal Institute with funds made available by the State of Illinois. The authors thank Wei Qiu at the State University of New York at Pittsburgh for providing chinchilla noise exposure and human hearing loss data. 


\section{References}

${ }_{330}$ [1] Y. Agrawal, E. A. Platz, J. K. Niparko, Prevalence of hearing loss and differences by demographic characteristics among us adults: data from the national health and nutrition examination survey, 1999-2004, Archives of Internal Medicine 168 (14) (2008) 1522-1530.

[2] M. Sliwinska-Kowalska, A. Davis, et al., Noise-induced hearing loss, Noise and Health 14 (61) (2012) 274.

[3] D. Henderson, R. P. Hamernik, Biologic bases of noise-induced hearing loss, Occupational Medicine-State of the Art Reviews 10 (3) (1995) 513-534.

[4] H. Crane, Mechanical impact: a model for auditory excitation and fatigue, The Journal of the Acoustical Society of America 40 (5) (1966) 1147-1159.

[5] G. R. Bock, E. J. Seifter, Developmental changes of susceptibility to auditory fatigue in young hamsters, International Journal of Audiology 17 (3) (1978) 193-203.

[6] B. C. J. Moore, An introduction to the psychology of hearing, Brill, 2012.

[7] J. C. Steinberg, M. B. Gardner, The dependence of hearing impairment on sound intensity, The Journal of the Acoustical Society of America 9 (1) (1937) 11-23.

[8] Acoustics - estimation of noise-induced hearing loss, Standard, International Organization for Standardization, Geneva, CH (Oct. 2013).

[9] U. D. of Health, H. Services, et al., Criteria for a recommended standard: occupational noise exposure. revised criteria 1998, Centers for Disease Control and Prevention, National Institute for Occupational Safety and Health.

[10] U. D. of Defense, Department of defense design criteria standard: Noise limits (mil-std-1474d). 
[11] M. H. Azizi, Occupational noise-induced hearing loss, The international journal of occupational and environmental medicine 1 (3 July).

[12] W. J. Murphy, C. A. Kardous, A case for using a-weighted equivalent energy as a damage risk criterion, National Institute for Occupational Safety and Health, EPHB Report No.

[13] P. Sun, J. Qin, W. Qiu, Development and validation of a new adaptive weighting for auditory risk assessment of complex noise, Applied Acoustics 103 (2016) 30-36.

[14] G. R. Price, Predicting mechanical damage to the organ of corti, Hearing research 226 (1) (2007) 5-13.

[15] W. J. Song, Study on human auditory system models and risk assessment of noise induced hearing loss, University of Cincinnati, 2010.

[16] A. Lopez-Najera, R. Meddis, E. A. Lopez-Poveda, A computational algorithm for computing cochlear frequency selectivity: Further studies, in: Auditory Signal Processing, Springer, 2005, pp. 14-20.

[17] N. Slepecky, Overview of mechanical damage to the inner ear: noise as a tool to probe cochlear function, Hearing research 22 (1) (1986) 307-321.

[18] B. Hu, Noise-induced structural damage to the cochlea, in: Noise-Induced Hearing Loss, Springer, 2012, pp. 57-86.

[19] G. R. Price, J. T. Kalb, Insights into hazard from intense impulses from a mathematical model of the ear, The Journal of the Acoustical Society of $375 \quad$ America 90 (1) (1991) 219-227.

[20] J. Deng, S. Chen, X. Zeng, G. Li, Using a dynamic tracking filter to extract distortion-product otoacoustic emissions evoked with swept-tone signals, Biomedical and Health Informatics, IEEE Journal of 18 (4) (2014) 11861195. 
[21] M. A. Ruggero, S. S. Narayan, A. N. Temchin, A. Recio, Mechanical bases of frequency tuning and neural excitation at the base of the cochlea: comparison of basilar-membrane vibrations and auditory-nerve-fiber responses in chinchilla, Proceedings of the National Academy of Sciences 97 (22) (2000) 11744-11750.

[22] L. Robles, M. A. Ruggero, Mechanics of the mammalian cochlea, Physiological reviews 81 (3) (2001) 1305-1352.

[23] P. Sun, J. Qin, K. Campbell, Fatigue modeling via mammalian auditory system for prediction of noise induced hearing loss, Computational and mathematical methods in medicine 2015.

[24] P. Sun, J. Qin, Auditory fatigue models for prediction of gradually developed noise induced hearing loss, in: 2016 IEEE-EMBS International Conference on Biomedical and Health Informatics (BHI), IEEE, 2016, pp. 384-387.

[25] M. C. Slama, M. E. Ravicz, J. J. Rosowski, Middle ear function and cochlear input impedance in chinchilla, The Journal of the Acoustical Society of America 127 (3) (2010) 1397-1410.

[26] M. Bance, F. M. Makki, P. Garland, W. A. Alian, R. G. Van Wijhe, J. Savage, Effects of tensor tympani muscle contraction on the middle ear and markers of a contracted muscle, The Laryngoscope 123 (4) (2013) 10211027.

[27] W. A. Ahroon, Effects of acoustic impulses on the middle ear, Tech. rep., DTIC Document (2015).

[28] B. Moore, B. Glasberg, A model of loudness perception applied to cochlear hearing loss, Auditory Neuroscience 3 (3) (1997) 289-311.

[29] P. A. Vrettakos, S. P. Dear, J. C. Saunders, Middle ear structure in the chinchilla: a quantitative study, American journal of otolaryngology 9 (2) (1988) 58-67. 
[30] R. L. Goode, M. Killion, K. Nakamura, S. Nishihara, New knowledge about the function of the human middle ear: development of an improved analog model., Otology \& Neurotology 15 (2) (1994) 145-154.

[31] J. J. Rosowski, The effects of external-and middle-ear filtering on auditory threshold and noise-induced hearing loss, The Journal of the Acoustical Society of America 90 (1) (1991) 124-135.

[32] R. Meddis, L. P. OMard, E. A. Lopez-Poveda, A computational algorithm for computing nonlinear auditory frequency selectivity, The Journal of the Acoustical Society of America 109 (6) (2001) 2852-2861.

[33] W. Rhode, N. Cooper, Nonlinear mechanics in the apical turn of the chinchilla cochlea in vivo, Auditory Neuroscience 3 (2) (1996) 101-121.

[34] A. J. Oxenham, C. A. Shera, Estimates of human cochlear tuning at low levels using forward and simultaneous masking, Journal of the Association for Research in Otolaryngology 4 (4) (2003) 541-554.

[35] W. M. Hartmann, Signals, sound, and sensation, Springer Science \& Business Media, 1997.

[36] R. P. Hamernik, W. Qiu, Energy-independent factors influencing noiseinduced hearing loss in the chinchilla model, The Journal of the Acoustical Society of America 110 (6) (2001) 3163-3168.

[37] R. P. Hamernik, W. A. Ahroon, Sound-induced priming of the chinchilla auditory system, Hearing research 137 (1) (1999) 127-136.

[38] Y.-m. Zhao, W. Qiu, L. Zeng, S.-s. Chen, X.-r. Cheng, R. I. Davis, R. P. Hamernik, Application of the kurtosis statistic to the evaluation of the risk of hearing loss in workers exposed to high-level complex noise, Ear and hearing 31 (4) (2010) 527-532.

[39] G. S. Goley, W. J. Song, J. H. Kim, Kurtosis corrected sound pressure level as a noise metric for risk assessment of occupational noises, The Journal of the Acoustical Society of America 129 (3) (2011) 1475-1481. 
[40] R. P. Hamernik, W. Qiu, B. Davis, Hearing loss from interrupted, intermittent, and time varying non-gaussian noise exposure: The applicability of the equal energy hypothesis, The Journal of the Acoustical Society of America 122 (4) (2007) 2245-2254.

${ }_{440}$ [41] K. Campbell, A. Claussen, R. Meech, S. Verhulst, D. Fox, L. Hughes, Dmethionine (d-met) significantly rescues noise-induced hearing loss: Timing studies, Hearing research 282 (1) (2011) 138-144. 